\title{
Über Trachoma verum corneae und sein Wesen.
}

\author{
Von
}

Dr. C. Pascheff,

Sofia (Bulgarien).

Mit Taf. XVI u. XVII, Fig. 1-31.

Eine der häufigsten Begleiterscheinungen des Trachoms ist der Pannus. Trotzdem bleiben die Anschauungen über seine Natur noch heute so verschieden wie früher.

So ist für einige Autoren der Pannus trachomatosus ein eigentlicher Teil des Trachoms: er ist ein Trachom der Hornhaut; fuir andere Autoren ist er, im Gegenteil, nur eine Begleiterscheinung des Trachoms (Muttermilch). Für einige wiederum zeigt er dieselbe Struktur wie die trachomatöse Bindehaut; während für andere er eine Keratitis rasculosa superficialis ist (Goldzieher).

Auch diejenigen, die behaupten, dass der Pannus trachomatosus von derselben Natur wie die trachomatöse Bindehaut ist, unterscheiden sich in der Anschaumg über seine histologische Struktur.

So enthält nach Rählmann und Addario der Pannus trachomatosus Follikeln, die nach Nuel ohne Gefässe sind; Ichikawa dagegen behauptet, dass er ein Granulationsgewebe ohne Follikeln, reich an Blutgefässeu und Zellen von zweierlei Art: Plasmazellen und Fibroblasten, ist.

Nach Bietti besteht der Pannus trachomatosus nicht nur aus einer rundzelligen Infiltration: dazwischen findet man auch zahlreiche Spindelzellen. Ausserdem sind Blutgefässe und Bindegewebe vorhanden. An einigen Stellen besteht dièses Bindegewebe aus feinen Fasern und bildet ein kleinmaschiges, mit rund- und spindelförmigen Zellen gefülltes Netz; an andern Stellen beobachtet man dicke Bündel, die wie sklerosiert aussehen.

Meyerhof hat die Follikelbildung in der Hornhaut, Pannus bei Trachom, beschrieben, hat aber keinen histologischen Beweis dafür 
erbracht, da er „kein geeignetes Material zur Anfertigung mikroskopischer Schnitte" gehabt hat.

Nach dem Buch von Prof. Stanculeanu und Michail über das Trachom nach dem gegenwärtigen Stand der Forschung entsteht der Pannus aus einer Neubildung des Bindehautgewebes, welches mit zahlreichen Lymphocyten infiltriert ist.

Letztens, nach dem soeben erschienenen Buch von Prof. Römer, „dürfen wir nicht erwarten, dass wir im Gewebe der Cornea dabei eine ausgesprochene Follikelbildung antreffen, weil ja die Vorbedingung für ihre Entstehung, der adenoide Gewebsbau, fehlt".

Der Sitz des Pannus trachomatosus ist ebenfalls unbestimmt: für einige liegt er unter der Membrana Bowmani (Rählmann), für andere - zwischen ihr und dem Epithel.

Aus dem oben Bemerkten ersieht man, welche grosse Verschiedenheit in der Auffassung der Struktur und Natur des Pannus trachomatosus heute herrscht.

Merkwürdig ist es, dass trotz so vieler Arbeit und Forschung dieselbe Verschiedenheit in der Anschauung der Natur des Trachoms sich noch heute fortsetzt.

So ist nach einigen, selbst nach den neuesten Untersuchungen, das Trachom eine diffuse entzündliche Wucherung der subconjunctivalen. Gewebsschicht; nach andern ist es eine hyperplastische Vordickung der adenoiden Schicht der Bindehaut mit Hervorbringung von FollikeIn. Dritte rechnen das Trachom zu den chronischen Entzündungen der Bindehaut und betrachten die Follikeln und den Pannus als Begleiterscheinung des Trachoms (Muttermilch).

Vierte betrachten das Trachom als proliferative Gewebsveränderung, die mit Wucherung von Plasma- und Bindegewebszellen einhergeht, und das Auftreten von Follikeln keineswegs von dem eigentlichen Trachomprozesse abhängt (Ichikawa).

Nach Saemisch wird als Conjunctivitis granulosa (Körnerkrankheit), diejenige Entzündungsform der Bindehaut bezeichnet, bei welcher in der adenoiden Schicht derselben eine entzündliche Infiltration auftritt, die von der Entwicklung von Follikeln (Granula, Körner), sowie von einer Wucherung des Papillarkörpers begleitet wird und unter Umwandlung der erkrankten Bindehautabschnitte in Narbengewebe abläuft.

Nach Goldzieher handelt es sich um eine in der Gefässschicht der Conjunctiva einsetzende, proliferative Entzïndung, mit Gruppierung der perivaskulären Zellinfiltrate zu knötchenförmigen 
Granulomen. Die Primitivknötcheı bestehen fast ganz aus Plasmazellen.

Stanculeanu und Michail, in ihrem hier oben citierten Buch, schreiben das folgende:

"Saemisch und nach ihm alle klassischen Autoren haben bei der adenoiden Schicht eine charakteristische Bildung, trachomatöses Follikel genannt, beschrieben. Wir miissen indessen bemerken, dass dieses histologische Aussehen viel seltener vorkommt, als die Autoren annehmen, sa dass der Follikel für das chronische Trachom durchaus nicht charakteristisch ist."

In einer Arbeit über das Trachom, die ich im Konkurs zu Budapest (1909) eingebracht und in den Archives d'ophtalmologie (Janvier 1910) veröffentlicht habe, bin ich zu den folgenden Schlüssen über die Natur des Trachoma chronicum verum gekommen:

"Le Trachome granuleux chronique simple, le seul vrai trachome, se caractérise par un tissu lymphoadénoïde à centres germinatifs isolés ou agminés;

Ce tissu, par sa structure, est semblable au tissu des granulations du pharynx et des végétations adénoïdes du nasopharynx et paraît être, très probablement, de la même nature."

In derselben Arbeit habe ich einige klinische and histologische Tatsachen über den Panuus trachomatosus dargelegt, die von grosser Bedeutung für uns hier sind. Seitdem hatte ich die Gelegenheit, noch viel mehr Material von Pannus trachomatosus zu studieren, was uns in die Lage bringt, ein neues Licht über diese so schwere und viel diskutierte Frage zu werfen.

Schon im Anfang meiner Untersuchungen über die Natur des Trachoms habe ich wahrgenommen, dass der Pamnus trachomatosus sich nicht immer zeigt als eine starke Vaskularisation der Hornhaut, aber in Form kleiner blasser graugelber Knötchen wie Follikeln besonders in der Nähe des oberen Limbus auftritt; nichtsdestoweniger war diese klinische Feststellung für mich noch nicht ausreichend, das Wesen des Pannus trachomatosus zu erklären; deswegen habe ich zum Mikroskop gegriffen und jeden Panuus, der möglich war zu excidieren, histologisch untersucht.

In folgenden Zeilen werde ich die Resultate dieser Untersuchungen in Kürze darlegen.

Beror ich die Beobachtungen vorbringe, sei es mir gestattet, einige Worte über die Technik zu sagen.

Ich habe bei der Operation den Schnitt in den meisten Fällen 
gegen die gebräuchliche Methode des Pterygium nicht von der Hornhaut aus, sondern von der Augapfelbindehaut getan. Alsdann erfasse ich die Bindehaut, ziehe sie nach unten und löse mit der Lanzette den Pannus von der Hornhant los. Es kommt vor, dass der Pannus mir am Limbus festhält und sich leicht von dem Rest der Hormhaut loslöst. Die Methode hat mir besser gedient, den Pannus ganz zu lösen, als diejenige, die man gewöhulich beim Pterygium anwendet.

Ich muss noch bemerken, dass ich für meine Studien nur die gut entwickelten Pannus (meistens unter dem Namen von Pannus carnosus und sarcomatosus bekaunt), die es mir gelungen ist, vollständig zu lösen, untersucht habe.

Die Biopsies waren in Alkohol absolut fixiert und gehärtet.

Als Farben habe ich besonders Giemsa (Romanowsky), Polychrommethylenblau (Unna) and Pyroninmethylengrün (Unna-Pappenheim) angewendet. Letztere habe ich besonders gut befunden, die Keimzentren von den umgebenden Plasmazellen zu unterscheiden.

In einigen Fällen habe ich auch den Inhalt des Pannus durch die Strichmethode studiert.

\section{Fall.}

Es handelt sich um einen 19 jährigen Bauern, der seit 4 Monaten an Trachona granulosum elrronicum verum leidet.

Rechtes Auge (Fig. 11, Taf. XVI): Der Limbus superior der rechten Hornhaut ist ziemlich verdickt, nnd zahlreiche Gefässe bedecken die obere Häilfte der letzteren und verästeln sich auf ilirer Oberfläche. Dicht an dem Limbus ist die Verdickung nicht gleichmässig, sondern zeigt mehrere kleine, blasse und gräuliche Knötchen, gegen welche sich sehr foine Gefässe riehten, die sich in den Knötchen verlieren. Diese kleinen Knötchen, die sehr deutlich nmgrenzt erscheinen, haben das Aussehen von echten Follikeln solche, die man gewöhnlich über der Bindehaut beobachten kann.

Die mikroskopische Untersuchung eines vertikalen Querschnittes durch die hier oben beschriebene follikuläre Verdickung an der Hornhaut zeigt das folgende (Fig. 24, Taf. XVI):

Schon bei schwacher Vergrösserung sieht man das Epithel an einigen Stellen stark abgeplattet und verdünnt, an andern verlängert sich dasselbe in das Gewebe. Dort, wo das Epithel ganz dünn ist, erkennt man kaum seine basalen Zellen, während dort, wo es sich verlängert, die Zellen deutjicher erseheinen und mehrere Lymphocyten und polynukleäre Leukocsten zeigen.

Das subepitheliale Gewebe zeigt eine adenoide Struktur und ist mit zahlreichen Zellen infiltriert. Unmittelbar unter dem Epithel befinden sich besonders die Plasmazellen, während tiefer die Lymphoeyten vorherrschen. Die letzteren sind in starker Proliferation vorhanden und zeigen alle transitorischen Formen der mitotischen und amitotischen Teilung. An einigen Stellen gruppieren sie sich zur Bildung von Knötchen, die den ecliten Fol- 
likeln ähneln, mit dem Untersehied, dass sie keine lymphocytäre Randzone aufweisen. Zwischen djesen Knötchen bemerkt man zahlreiche Plasmazeilen.

Das Gewebe ist mit einer grossen Anzahl von Gefässen durehzogen die in starker Proliferation sind.

\section{Fall.}

Es handelt sich um ein 16 jähriges Mädchen, welches sein 3 Jahren an Trachoma granulosum ehronicum verum leidet.

Linkes Auge (Fig. 15, Taf. XVI): Die Bindehaut des oberen Fornices ist noch mit zahlreichen Granulationen bedeckt.

Auf der inneren Hälfte der Conjunctiva sclerae bemerkt man ein ziemlich grosses Gefäss, das von der Plica semilunaris ausgeht, horizontal nach der Hornhaut zu läuft und sich auf der oberen Hälfte der Hornhaut verästelt.

Auf der letzteren, zwischen diesem grossen Gefäss und dem oberen Limbus, bildet sich ein gräuliches, gefässreiches Häutchen, das sich nach dem Limbus zu verdickt, wo es einige sehr deutlich umgrenzte Follikeln aufweist.

Auf einem vertikalen Quersehnitt desselben zeigt sieh folgendes:

Das Epithel erseheint stark abgeplattet und geschichtet, besonders da, wo das Gewebe am stärksten infiltriert ist. An andern Stellen ist es ziemlich verdickt und zeigt einige polynukleäre Leukocyten.

Das subepitheliale Gewebe hat eine adenoide Struktur, reich an Gefässen, deren Wände stark proliferierend sind. Das Bindegewebe ist verschiedenartig entwickelt: an einigen Stellen ist es sehr verdickt, an andern ist es durch zahlreiche, stark proliferierende Lymphocyten ersetzt. Die letzteren haben sich stark angesammelt und bilden echte Keimzentren. Zwischen diesen, in der Umgebung der grossen Gefässe, und unmittelbar unter dem Epithel befinden sich besonders viel Plasmazellen, die oft eine hyaline Degeneration zeigen.

\section{Fall.}

Es handelt sich um einen 20jährigen Mann, der seit einem Jahre an Trachoma granulosum chronicum verum leidet.

Rechtes Auge (Fig, 25, Taf. XVII): In der oberen Hälfte des Bulbus bemerkt man eine starke Injektion der Bindehautgefässe, die nach dem Limbus superior zu verlaufen und tiber die Hornhaut sich verästeln. Ganz dicht an dem Limbus zeigt sich eine deutliche Verdickung, auf welcher man die stark ausgeprägten Silhouetten mehrerer Follikel wahrnimmt. Diese Follikel sind glatt, gefässreicl, granlich gefärbt und von der Grösse eines Hirsekornes und grösser. Die Bindehantgefüsse, die nach diesen Follikeln zu verlaufen, versehwinden in ihrem Gewebe, um dann wieder herauszutreten und Halbkreise zu bilden, von denen ans feine Gefässehen sich nach den Follikeln zu erstrecken, wo sie sich über deren Oberfläche verbreiten und sich dann verlieren.

Die mikroskopische Untersuchung eines vertikalen Quersehnittes ergibt folgendes:

Schon bei schwacher Vergrösserung sehen wir das Epithel an einigen 
Stellen verdickt and deutlich, an andern Stellen ist es stark verflacht und geschichtet, und zwar so, dass man nur mit Mühe die basalen Zellen erkennen kann.

Dort, wo das Epithel am stärksten geschichtet ist, ist das subepitheliale Gewebe auch am stärksten infiltriert.

Das subepitheliale Gewebe hat eine adenoide Struktur. Die Gefässe sind stark proliferierend. Das ganze Gewebe ist mit zahlreichen Lymphocyten infiltriert, welche sich anhäufen und durch ihre starke Proliferation echte Keimzentren bilden. In der Umgebung der letzteren findet man viele kleinere Lymphocyten und Plasmazellen, aber ohne dentliche lymphocytäre Zone. Die Plasmazellen treten in der Cmgebung der grösseren Gefässe und besonders unter dem Epithel auf, wo sie die sogenannte plasmacelluläre Schicht bilden.

\section{Fall.}

Es handelt sich um ein 13jähriges Mädchen, das an beiden Augen an Trachoma granulosum chronieum verum mit zahlreichen Granulationen Jeidet.

Rechtes Auge (Fig. 28, Taf: XVII): Die Bindehautgefässe, die von der oberen Fornix ausgehen und sich nach der Hornhaut erstrecken, wo sie sich verästeln, sind stark injiziert. Die ganze obere Hälfte der Hornhaut ist mit einem trüben, gräulichen, gefässreichen Schleier bedeckt. In der Nähe des Limbus superior verdickt sich der Schleier stark, und man gewahrt eine Reihenfolge von kleinen, gräulichen Knötchen, die das Aussehen von echten FFollikeln haben. Über die letzteren verbreiten sich zahlreiche feine Gefässe.

Auf den mittleren Teilen der Hornhaut sieht man hier und dort kleine Flecken von schmutzig weisser Färbung, welche um so deutlicher erscheinen, je durehsichtiger die Hornhaut ist.

Bei der mikroskopischen Untersuchung eines vertikalen Querschnittes bemerkt man folgendes:

Das Epithel ist stark verflacht und geschichtet. An einigen Stellen ist es fast unerkennbar, während an andern, obgleich stark infiltriert, man deutlich einige Schichten unterscheiden kann.

Unmittelbar unter dem Epithel zeigen sich, infolge der Färbung mit Pyroninmethylengrün, zahlreiche Plasmazellen. Unter dieser plasmacellularen Schicht verschwindet das Bindegewebe, die Gefässe lösen sich in kleine Kapillaren auf, und die ganze Masse ist mit zahlreichen, stark proliferierenden Lymphocyten infiltriert - denselben, die man in den Keimzentren der Follikel findet. Kurz, diese ganze Masse erscheint uns wie eine trachomatöse Granulation, die sich durch den Zusammenffuss melrerer kleiner Keimzentren gebildet hat.

Linkes Auge: Hier ist der Schleier, welcher die obere Hälfte der Hornhaut bedeckt, noch stärker entwickelt. Nahe am oberen Limbus hin unterscheidet man deutlich die Silhouetten zahlreicher Follikeln.

Die mikroskopische Untersuchung zeigt uns:

Das Epithel hat ungefähr dieselben Veränderungen erlitten wie dasjenige des rechten Auges.

Das subepitheliale Gewebe lässt uns schon bei schwacher Vergrösserung 
grosse cellulare Anhäufungen exkennen, welche las Aussehen von echten Follikeln haben. Einige sind rund, andere länglich. Die cellularen Anhänfungen sind durch Bindegewebsstrecken und einige epitheliale Verlängerungen getrennt (Fig 29, Taf. XVII).

Bei einer stärlieren Vergrösserung sieht man, dass infolge der Färbung mit Pyroninmethylengrün zahl'eiche Plasmazellen unter dem Epithel und in der Umgebung der epithelialen Verlängerungen hervortreten.

Unter der plasmacellularen Schicht bestehen die oberen cellularen Anhäufungen ausnahmsweise aus Lymphocyten. Die letzteren weisen eine grosse Proliferation auf und bilden echte Keimzentren, welche teilweise ron lymphocytärer Zone umgeben sind.

Nach der Peripherie zu gehen einige von diesen Keimzentren uber in Cieatrisation und zeigen die charakteristischen, fadenartigen Elemente, denen man oft in den adenoiden Geweben begegnet.

\section{Fall.}

Es handelt sich nm einen 28 jährigen Bauern, der an Trachoma chronicum verum gelitten hat, und dessen Bindehaut zallreiche Narben zeigt. (Fig. 19, Taf. XVL.)

Linkes Auge: Die sklerale Bindehaut, vor allem oben und in der Nahe des Limbus superior, ist stark injiziert ber Limbus superior und fast die ganze obere Hälfte der Hornhaut ist bedeckt mit einer geschwulstartigen Masse von rotweinälnlicher Farbe, mit deutlich begrenztem Rande, der nach der Hornhaut zu plötzlich steil abfält. Die Oberfäche ist glatt, aber zeigt hier und da einige kleine Buckel. Diese geschwulstartige Masse ist sehr gefässreich, blutet leicht und ist von weicher Konsistenz. Zahlreiche Gefässe von der oberen slileralen Bindehaut laufen nach dieser Masse za, wo einige derselben dort verschwinden, während andere sich in zahlreiche Ästchen verzweigen, an der Oberfäche bleiben und dieselbe umgeben. An dem oberen Rande der Masse bemerkt man kleine vertikal verlängerte, blassgraue, durch Gefässe voneinander getrennte Follikeln, die das Aussehen von echten Follikeln haben. Unter dem unteren Rande der Masse zeigen sich einige kleine Geschwüre. In der Nähe des Limbus internus sieht man einige grosse, gefässreiche Knötchen, die viel an echte trachomatöse Follikeln erinnern.

Die mikroskopische Untersuchung ergibt:

1. Auf einem vertikalen und parallel zu dem vertikalen Meridian der Homhaut laufenden Querschnitte, zeigte die äussere Hälfte dieser Masse oder Geschwulst einen dünneren und einen dickeren Teil. Der dünne Teil, der $1 \mathrm{~mm}$ lang ist, ist die sklerale Bindehaut selfst; der dicke Teil ist derjenige, der auf der Hornhaut liegt (Fig. 26, Taf, XVID).

a) Der dünne Teil. Das Epithel, welches den dünnen Teil bedeckt, ist stark abgeplattet und geschichtet, derart, dass es kaum sichtbar ist. Es befindet sich nux ganz nahe an der Geschwulst, wo es eine dreiziptlige Verlängerung in das subepitheliale Gewebe linein schiebt, und wo die epithelialen Zellen deutlicher erscheinen.

Die adenoide Schicht der Bindelaut ist ziemlieh verdickt, gefässreich und stark infiltriert mit Plasmazellen, von denen einige hyaline Degeneration erleiden und melrere Rüsselsche Körperchen zeigen. Neben den 
Plasmazellen befinden sich auch Lymphocyten, die besonders an zwei Stellen sieh vereinigen und echte Keimzentren bilden.

b) Der dicke Teil. Das Epithel ist noch stärker geschichtet und unerkennbar. Es ist nur an dem untersten der Geschwulst besser erhalten und deutlicher. Hier findet man die Bowmansche Membran stark aufgelöst und gefaltet. Infolge der Färbung mit Pyroninmethylengrün bemerk man hauptsächlich zweierlei Zellen, die die ganze Geschwulst ausfïllen, Hiervon sind einige Plasmazellen und andere Lymphocyten. Die Plasmazellen befinden sich meistens unter dem Epithel, weil sie im Gewebe der Geschwulst weniger und zerstreut vorhanden sind.

Die Lymphocyten dagegen erfülten fast die ganze Geschwulst. Sie zeigen alle Formen der mitotischen und amitotischen Teilung und bilden grosse unbegrenzte, meistens vereinigte Keimzentren, die stellenweise durch Bindegewebsstreifen getrennt sind.

Die Gefässe der Geschwulst sind stark proliferierend. Das Bindegewebe ist nur hier und da etwas entwickelt, und zwar mehr an der Basis des Tumors.

2. Fig. 27, Taf. XVII. Auf einem horizontalen und parallel zu dem horizontalen Meridian der Hornhaut laufenden Querschnitt zeigt die innere Hälfte dieser Geschwulst dieselbe Struktur und dieselben Zellen. An den dicksten Teilen des Tumors bemerkt man in dem letzteren einzelne Läppchen, welche dureh kleine Zwisehenräume und grössere Gefässe getrennt sind. Eins von diesen Läppchen erscheint ganz isoliert und zeigt an der Peripherie zahlreiche fadenförmige Elemente.

Die Läppchen bestehen fast ausschliesslich aus blassen, grossen Lymphocyten - denselben, denen man in den Keimzentren begegnet.

An einigen Stellen sielit man unter dem Epithel einen grossen Teil der Bow m a n schen Membran aufgelöst und zusammengerollt (Fig. 27a, Taf. XVII).

\section{Fall.}

Es handelt sich um eine 60jährige alte Frau, welche seit 16 Jahren an Trachoma chronieum leidet und niemals behandelt worden ist.

Die obere Hälfte des Bulbus zeigt eine ziemlich grosse, verbreitete Verdickung, welche fast die ganze obere Hälfte der Hornhaut bedeckt und sich über die sklerale Bindehaut, $5 \mathrm{~mm}$ über dem Limbus superior, erstreckt. Diese-Verdiekung ist von einer rötlichgrauen Färbung und hat eine unebene Oberfläche, auf welcher man die Silhouetten zahlreicher Follikeln erblickt. Sie ist sehr getässreich, und die Gefässe schicken nach den Follikeln mehrere kleine Abzweigungen, die sich dort verlieren (Fig. 18, Taf. XVI).

Die mikroskopisehe Untersuchung zeigt uns (Fig. 30, Taf. XVII):

Auf einem vertikalen und parallel za dem vertikalen Meridian der Hornhaut laufenden Quersehnitt sieht man, dass das Epithel der skleralen Bindehautverdiekung ziemlich gut erhalten ist. In der Nähe der Hornhaut und auf derselben ist es stark abgeplattet, geschichtet und fast unerkennbar. In den noch sichtbaren Teilen erseheinen die Zellen ödematös, und das Epithel ist mit sehr vielen Lymphocyten infiltriert.

Das subepitheliale Gewebe der skleralen Bindehautverdickung weist eine adenoide Struktur auf und ist reich an Plasmazellen. Diese plasma- 
cellulare Schicht nimmt nach der Hornhaut zu allmählich ab; dagegen nehmen die Lymphocyten zu. Das Bindegewebe, ebenso wie die Plasmazellen erleiden eine hyaline Degeneration.

In der Nähe der Hornhant wächst die Verdickung, das Bindegewebe nimmt $a b$, die Plasmazellen werden seltener und werden durch zahlireiche Lymphocyten ersetzt. Die letzteren sind stark proliferierend und zeigen alle typischen Formen, denen man im Keimzentren begegnet. Sie bilden grosse, ohne lymphocytäre Randzone agminierte Follikeln oder die sogenannten trachomatösen Granulationen. In den letzteren versehwindet das Bindegewebe fast und ist meistens durch das adventitielle Gewebe ersetzt.

An einigen Stellen werden die Lymphocyten seltener; und zwischen ihnen bemerkt inan mehrere makrophage und fadenförmige Elemente,

Wir haben den cellularen Inhalt dieser Verdickung mit der Strichmethode studiert und gefunden, dass er derselbe ist wie in den wahren trachomatösen Granulationen.

In der Tat sind die grossen Lymphocyten oder Keimzellen dieselben, die man gewölnlich in den Keimzentren der Follikeln antrifft. Im Verhältnis zu den kleinen Lymphocyten besetzten die ersten die tieferen Teile der Verdickung, während man die letzteren zwischen denselben und den Plasmazellen findet.

In dem dieksten Teil der Verdickung sind die Keimlymphocyten derartig zahlreich, dass sie ein grosses Keimzentrum bilden mit mehreren leeren Räumen und teilweise von einer lymphocytären und plasmacellulären Zone umgeben sind. Die Gefässe sind stark proliferierend.

An andern Schnitten erseheint dieses grosse Keimzentrum wie in zwei Teile dureh Gefässe und Bindegewebe getrennt. Gleiche Keimzentren findet man auch in dem zentralen Teile dieser Verdickung, aber kleiner und wenig entwickelt.

\section{Fall.}

Es handelt sich um einen Mann von 36 Jalıren, der an Trachoma granulosum elronicum leidet.

Das linke A uge zeigt mehrere kleine Follikel auf dem Tarsus superior, während der Fornix superior grosse Granulationen aufweist. Die letzteren sind weniger entwickelt in den Fornix inferior.

Am Limbus superior ist der sklero-corneale Winkel überrasehend deutlich ausgeprägt. Die Bindehantgefässe der oberen Hälte des Bulbus sind stark entwickelt und verlaufen nach der Hornhaut zu, die mit einem dicken, gräulichen, ungleichen, gefässreichen und geschwulstartigen Häutchen bedeckt ist. Die Gefässe laufen nach der Hornhaut zu in parallelen Reihen und teilen sich in zahlreiche Ästehen. Ganz nahe am Limbus superior, zwischen den grösseren Gefässen, wo das Häutchen am dicksten erseheint, heben sieh die Silhouetten wahrer, gefässreicher Follikel ab, die alle in einer Reihe liegen.

Das rechte Auge zeigt dieselben anatomischen Veränderungen, allein in einem weniger entwickelten Grade.

Die mikroskopisehe Untersuchung eines parallel dem horizontalen Me. ridian der Hornhaut laufenden Qnerschnittes lehrt uns: 
Schon bei schwacher Vergrösserung erscheint das Epithel ganz unregelmässig: an einigen Stellen ist es verdickt und mit Sprossen versehen, an andern ist es verdünnt und stark geschichtet.

Das subepitheliale Gewebe ist ron verschiedener Stärke, reich an stark proliferierenden Gefässen und zeigt überall eine adenoide Struktur.

Unmittelbar unter dem Epithel herrsehen die Plasmazellen vor; während man tiefer die Lymphocyten antrift. Die letzteren zeigen grosse Proliferation und bilden eelite Keimzentren mit lymphocytärer Zone versehen und ganz durchdrungen von dünnen Gefässchen.

Auf einigen Schnitten erscheinen die Keimzentren isoliert, auf andern schliessen sie sich zusammen und bilden durch leere Räume getrennte Läppchen, die wiederum reich sind an fadenförmigen Elementen.

Bei einigen Präparaten sieht man, wie in der Nähe des oberen Limbus die Follikeln an Ausdelnung zunebmen; bei andern - herrschen das Bindegewebe und die Plasmazellen vor. Dort, wo das Bindegewebe stark entwickelt ist, bemerkt man zahlreiche Mastzellen.

\section{Fall.}

Es handelt sich um eine Frau von 28 Jahren, welche an Trachoma seit 3 Jahren leidet, und deren Bindehaut zahlreiche Narben aufweist.

Oberhalb des Limbus superior ist die sklerale Bindehaut stark injiziert; nahe an dem Limbus beobachtet man eine grave Verdickung, die die obere Hälfte der Hornhaut bedeckt und sich mit der Entfernung verdünnt. Sie ist stark vaskularisiert, und auf ihrer Oberfläche, hauptsächlich in der Nähe des Limbus superior, sieht man eine grosse Anzahl von Follikeln (Fig. 17, Taf. XVI).

Die histologische Untersuchung eines perpendikulären und parallel zum horizontalen Meridian der Hornhaut lanfenden Schnittes durch die Oberfläche ergibt (Fig. 23, Taf. XVI):

Schon bei schwacher Vergrösserung sieht man, dass die dicksten Teile das Aussehen von echten Follikeln haben. Auf verschiedenen Schnitten ersoheinen diese Follikeln teils isoliert, teils vereinigt.

Das Epithelium, welches die dicken Teile bedeckt, ist stark verdünnt und geschichtet, während dasjenige, welches die weniger infiltrierten Teile bedeckt, deutlicher erscheint und melirere Sprossen in das subepitheliale Gewebe sendet. Es zeigt hier und da einige Lymphocyten und polynukleäre Leukocyten.

Die subepitheliale Schicht hat eine adenoide Struktur, ist reich an Gefässen und infiltriert mit Plasmazellen und besonders mit Lymphocyten. Die letzteren häufen sich an und bilden echte Keimzentren. Auf Serienschnitten bemerkt man, dass mit der Entfernung von dem Limbus die Gefässe im Durchmesser abnehmen, das Bindegewebe verschwindet, die Keimzentren grösser werden und sich vereinigen und grosse lymphoide Geschwülste bilden.

Die Mastzellen sind selten; noch seltener sind die eosinophilen Zellen.

Die Plasmazellen findet man meistens unter dem Epithel und zwischen den Keimzentren. Sie erleiden, ebenso wie das Bindegewebe, die hyaline Degeneration.

An einigen stellen bemerkt man zahireiche fadenförmige Elemente, die man in jedem lymphoiden Gewebe antrifft. 


\section{Fall.}

Es handelt sich um einen 20jährigen jungen Mann, der im zweiten Jahre seiner Militärzeit bemerkt, wie seine Augen schwächer werden.

Die ganze obere Halfte der rechten Hornhant ist mit einem graulichen Schleier, der stark vaskularisiert ist, bedeckt. Dieser Schleier bat eine etwas unebene Oberfläche und zeigt mehrere Follikeln in der Nähe des Limbus superior (Fig. 20, Taf. XVI).

Bei histologischer Untersuchung eines Sehnittes durch die Oberfläche, welcher parallel dem horizontalen Meridian der Hornhant verläuft, sieht man:

Das Epithel erscheint ganz geschichtet. Das subepitheliale Gewebe ist stark durch zweierlei Zellen infiltriert: unmittelbar unter dem Epithel herrschen die Plasmazellen vor; tiefer sind es zahlreiche Lymphocyten. Dio letzteren häufen sich an und bilden echte Keimzentren, die das Bestreben haben, sich zu vereinigen und grössere Granulationen zu bilden - die sind meistens in der Mitte der Schnitte zu finden.

Das Bindegewebe ist reich an Mastzellen. Die polynuklearen Leukocyten sind selten und treten meistens nur in dem Lumen der Gefässe auf.

Visus oculi dextri ror der Operation $=3 / 50^{\circ}$

$$
" \quad " \text { nach } " \quad, \quad=\left.6\right|_{20} ^{\circ}
$$

\section{Fall.}

Es handelt sich um einen Mann im Alter von 35 Jahren, welcher seit seinem 15. Lebensjahre an Trachoma chronicum leidet.

Das linke Auge zeigt folgendes Wiehtige:

Die Granulationen der Bindehaut sind durch zahlreiche Narben orsetzt. Das obere Lid ist verdickt, gewölbt, und viele Cilien reiben sich an der Hornhaut.

Nach dem oberen Limbus zu verlaufen zahlreiche Gefässe, welche von dem oberen Fornix kommen und sich auf der Hornhaut verästeln.

Oberhalb des Limbus und auf demselben verlieren sich die Gefässe in einer leicht graugelben Verdickung, auf welcher sich die Silhouetten kleiner Follikeln abheben.

In der Mitte der Hornhaut wird die Verdickung schwächer, und die feine Verzweigung der Gefässe erscheint deutlicher.

Bei der histologischen Untersuchung eines perpendiknlären Schnittes, weleher parallel zum horizontalen Meridian der Hornhaut verläuft, sehen wir:

Das Epithel ist an einigen Stellen ziemlich gut erhalten und zeigt etliche Lymphocyten, emigrierte Mastzellen und Pigmentzellen; auf andern ist es verdünnt und stark geschichtet. Unmittelbar unter dem Epithel herrschen die Plasmazellen vor.

Unter dieser plasmacellularen Schicht und nach der Mitte des Schnittes zu nimmt die lymphocytäre Infiltration za und bildet echte Keimzentren, nugeben von einer lymphocytären Zone von verschiedener Dicke. An dieser: Stelle ist das Epithel ganz verdünnt, geschichtet und fast unerkennbar.

An der Basis des Schnittes sind die Gefässe umbüllt mit zahlreichen Plasmazellen; das Bindegewebe ist reich an Mastzellen. 


\section{Fall.}

Es handelt sich um einen 35 Jahre alten Bauern, der seit 15 Jahren an Trachom leidet. Sein Trachom ist sehon in das Stadium cieatriceum eingetreten (Fig. 13, Taf. XVI).

Auf dem oberen Limbus bemerkt man folgendes: Die obere Hälfte der skleralen Bindehaut ist gefässreich. Diese Gefässe gehen von der oberen Fornix nach dem Limbus superior zu. Einige von ihnen bilden oberhalb des Limbus superior eine Kurve, von welcher zahlreiche Gefässchen nach der Hornhaut zu verlaufen. In der Mitte des Limbus superior sieht man eine grosse follikelähnliche Verdickung. Dieselbe ist stark vaskularisiert, dentlich hervorragend, von einer gräulichen Farbe und von ovaler Form, deren grosse Achse vertikal verläuft. Neben diesen grossen Follikeln zeigen sich auf der Hornhaut zwei andere Follikeln, die viel kleiner und ausserdem sehr undeutlich sind. Unterhalb dieser Follikeln erscheinen die Gefässe deutlicher und erreichen ansschliesslich die Mitte der Hornhaut. Die letztere zeigt mehrere Gefässe, die von allen Seiten des Limbus herzuströmen.

Auf einem mikroskopischen Schnitte, der parallel zum Limbus superior verläuft, sieht man, dass das Epithel an den dicksten Teilen stark abgeplattet ist, während es an den weniger infiltrierten Teilen ziemlich dünn erseheint (Fig. 22, Taf. XVI).

Unter dem Epithel, in der Mitte des Schnittes, bemerkt man ein grosses Keimzentrum. An der Basis desselben trifft man grosse Gefässe an. Auf Serienschnitten findet man, dass neben den grossen Follikeln sich andere, viel kleinere bilden.

Durch die Färbung mit Pyroninmethylgrün erscheint das subepitheliale Gewebe hauptsächlich von zwei Arten von Zellen inflitriert.

Die einen, die die Keimzentren bilden, sind die Lymphocyten; die andern - mit stark rotgefärbtem, randständigem Protoplasma - sind die Plasmazellen. Die letzteren finden sich meistens in der Umgebung der Gefässe und vor allem unter dem Epithel, wo sie die plasmacellulare Schicht bilden. Es ist eine eigentümliche Erscheinung, dass dort, wo die Follikeln am stärksten entwickelt sind, nicht "inur das Epithel dünner wird, sondern anch die plasmacellulare Schicht, die zwischen dem Epithel und den Follikeln liegt.

Die Keimzentren sind von keiner Iymphocytären Zone nmgeben, aber sie enthalten dieselben cellularen Elemente, wie die typischen Follikeln. Sie sind von zahlreichen kleinen Gefässchen durehdrungen und zeigen an einigen Stellen kleine leere Räume, die mit Blut und Fibrine gefüllt sind.

\section{Fall.}

Es handelt sich um einen 16jährigen Bauern, welcher an Trachoma chronicum seit 4 Jahren leidet.

Rechtes Ange (Fig. 16, Taf. XVI):

Von der skleralen Bindehaut gehen zahlreiche Gefässe nach der Hornhaut zu und verzweigen sich auf deren Oberfläche. Einige von diesen Gefässen bleiben ganz an der Oberfläche; andere befinden sich weiter hinten, erseheinen blass auf der Sklera und werden deutlicher auf der Hornhaut. 
An ihrem oberen Drittel zeigt die Hornhaut eine graugefärbte Verdiekung, welehe stark vaskularisiert ist und an dem oberen Limbus beginnt. Die Verdickung ist nicht gleichmässig, sondern zeigt mehrere Knötchen. Diese sind durch grössere Gefässe getrennt. Thre Oberfläche ist ziemlich hervorstehend and hat das Aussehen von echten Follikeln. Von den grösseren Gefässen, die zwischen den Knötchen liegen, gehen kleinere, sebr zarte aus, die sich über die Oberfläche jedes Follikels verbreiten und dann versehwinden.

Die mikroskopisehe Untersuchung eines parallel zum Limbus superior laufenden Schnittes zeigt uns, dass da, wo das subepitheliale Gewebe dick ist, das Epithel ganz verdiunnt erscheint, und umgekehrt.

Das subepitheliale Gewebe ist arm an Bindegewebe, aber reich an Zellen. Diese sind hauptsächlich Lymphocyten, die grosse, zusammenfliessende Keimzentren bilden. Die Plasmazellen sind seltener und finden sich unter dem Epithel, zwischen den Keimzentren und in der Umgebung der grösseren Gefässe, wo das Bindegewebe mehr entwiekelt erscheint. Die Gefässe sind stark proliferierend.

\section{Fall.}

Es bandelt sich um eine 30jahrige Frau, die seit 4 Jahren an Trachoma chronicum leidet.

Rechtes Auge. Die Bindehaut des Tarsus superior und inferior zeigt zahlreiche Cicatricen. Der untere Fornix ist stark verkürt. Die Conjunotiva sclerae, besonders diejenige der oberen Hälfte des Bulbus ist stark injiziert, hauptsächlich in der Nähe des Limbus superior. Die Gefässe gehen über den Limbus und verästeln sich über die Hornhaut. Die Conjunctiva selerae, ungefähr $3 \mathrm{~mm}$ über den Limbus superior verdickt sich und zeigt in der Mitte einen echten Follikel (Fig. 14, Taf. XVI).

Die Hornhaut ist in ibrer oberen Hälfte stark oberfächlich vaskularisiert und zeigt eine grane Trübung mit zallreichen kleinen Gefässen.

Die mikroskopische Untersuchung des rerdickten Teiles der Conjunctiva sclerae, nicht weit vom Limbus superior, nämlich dort, wo der Follikel liegt, zeigt:

Schon bei einer schwachen Vergrösserung sieht man, dass in der Mitte des Präparates die subepitheliale Infiltration verdickt ist und einen echten Follikel bildet (Fig. 21, Taf. XVI).

Bei stärkerer Vergrösserung sieht man, dass der verdickte Teil des Präparates einen typisehen Follikel mit Keimzentrum zeigt, und die dünnere cellulare subepitheliale Infiltration reicher ist an Plasmazellen und Fibroblasten.

Das Epithel über dem Follikel ist stark gesehichtet, uber den wenigen infiltrierten Teilen ist es deutlicher und zeigt mehrere emigrierte Lymphocyten, Mastzellen und einige Pigmentzellen.

Die Gefässe sind uberall in starker Proliferation.

XIV. Fall.

Es handelt sich um einen 32 jährigen Bauern, der seit 12 Jahren an Trachoma verum chronicum leidet.

Rechtes und linkes Ange: Die Bindehant ist überall stank gerötet, 
mit vielen, im Stadium cicatriceum übergehende Granulationen versehen, die deutlicher in den unteren und oberen Fornix erscheinen.

Die Hornhaut zeigt einen stark entwickelten Pannus vaseulosus mit rauher Oberfläche. An der inneren Hälfte des Balbus verdickt sich die Bindehaut und bildet einen Psendopterygium, der mit der Spitze auf der Hornhaut klebt. Die Oberfläche dieses Pterygiums ist ungleich und zeigt mehrere deutliche Follikeln (Fig. 31, Taf. XVI).

Die histologische Untersuchung dieses Pterygiums zeigt eine starke lymphocytäre Infiltration der subepithelialen Gewebe mit einigen deutlichen Follikeln.

Indern wir die hier oben zusammengefassten Beobachtungen studieren, sehen wir schon klar und deutlich, dass das Trachoma verum chronicum der Bindehaut oft ron einigen bestimmten Bildungen auf der Hornhaut begleitet ist, welche man früher unter den allgemeinen Namen „Pannus" gekannt hat, und die histologisch noch sebr wenig untersucht sind.

Diese cornealen Bildungen erscheinen klinisch in der Form von Follikeln, Granulationen und geschwulstartigen Verdickungen. Sie sind dieselben, die man auf der Bindehaut bei Trachoma verum chronicum beobachtet. Diesen Bildungen auf der Hornhaut geben wir den Namen "Trachoma verum corneae".

Also betrachtet, ist das Trachoma verum corneae nicht mehr ein einfacher "Pannus", sondern eine Bildung, die differenzierter und komplizierter ist.

Diese besondere Bildung entwickelt sich spontan und hat einen chronischen Verlauf. Sie ist nicht nur am Limbus begrenzt, sondern geht auch über denselben hinweg auf die Hornhaut und merkwürdigerweise auch auf die sklerale Bindehaut. Diese Tatsache, dass sich das Trachoma verum nicht nur vom Limbus nach der Hornhaut zu fortpflanzt, sondern auch auf die sklerale Bindehaut, haben wir in unserer Arbeit uiber das Trachoma verum conjunctivae schon mitgeteilt. Es ist so selten und nirgends wird in der Literatur davon geredet, so dass einige Autoren sich mit grossem Recht gefragt haben: "Warum greift vom Limbus aus die Entzündung auf die Hornhaut und nicht umgekehrt auf die Conjunctiva sclerae über?"

Diese Frage scheint uns von grosser Wichtigkeit zu sein; denn seine Beantwortung wirft nenes Licht in die Natur des Trachoma verum der Bindehaut selbst. Es handelt sich jetzt darum, zu wissen, ob das Trachoma verum im Wesen eine einfache Entzündung ist oder eine besondere Hyperplasie.

Die meisten der heutigen Autoren betrachten das Trachom als

v. Graefe's Archiv für Ophthalmologie. LXXXVII. 3 . 
eine Entzündung und finden die Antwort in der zentripetalen Richtung der Blutcirkulation in der Conjunctiva sclerae.

Diese Erklärung ist ausgezeichnet, wenn man das Trachoma verum als einen einfachen inflammatorischen Prozess betrachtet; aber sie ist ungeniigend durch die nenen klinischen Tatsachen, welche wir festgestellt haben, nämlich, dass die trachomatösen Follikeln und Granulationen, die charakteristische fuir das Trachoma verum sind, sich nicht nur am Limbus bilden, sondern sich in derselben Zeit über die Hornhaut und die Conjunctiva sclerae fortpflanzen können.

Diese neue Tatsache zeigt uns, dass das Trachoma verum corneae nicht eine einfache Entzündung, noch eine einfache Gefässneubildung ist, sondern etwas mehr - nämlich - eine Hyperplasie. Dies ist ein neues Gewebe - wix lernen es später kennen bei der Besprechung seiner Struktur und Entstehung -, welches in der Umgebung der existierendẹn Gefässe und ïberall da, wo sich neue Gefässe bilden, herrscht und in eine üppige Vegetation übergeht.

Die Struktur dieses Gewebes weist noch weitere charakteristische Züge auf, die es von den andern „Pannus" unterscheidet.

Daraufhin haben wir mehrere Fälle von Pannus studiert: Pannus lymphaticus, Pannus trachomatosus, Pannus bei Conjunctivitis vernalis und Plasmome der Hornhaut.

Im folgenden werden wir kurz zusammenfassen, was wir in den Versuchen festgestellt haben.

Beim Frühjahrskatarrh ist der Pannus reich an fibrösen Bindegeweben. Die cellulare Infiltration, die hauptsächlich in Lymphocyten und Plasmazellen besteht, wie wir in unserer Arbeit über Frühjahrskatarrh gezeigt haben (Arch. d'opht. 1907), hört am Limbus auf, und nur einmal habe ich Follikel in der fibrösen perilimbären Verdickung beobachtet.

Bei gewissen Fällen ron Pannus lymphaticus können sich starke, rötliche und fleischige Verdickungen bilden, die histologisch eine adenoide Struktur aufweisen, durch Lymphocyten, einige polynukleäre Leukocyten und zahlreiche Plasmazellen infiltriert.

Bei andern - viel selteneren Fallen - haben wir geschwulstartige Bildungen der Hornhaut mit zahlreichen proliferierenden Plasmazellen beobachtet, die wir als Plasmome der Hornhaut') beschrieben haben.

Bei allen diesen Fällen von Pannus sehen wir, dass sie histo-

1) Diese sind hente durch Rund, Pokrowsky, Schikau und Deatschmann für die Bindhaut bestätigt worden. Zeitschr. f. Augenheilk. 1912. 
logisch reich an Plasmazellen und polynuklearen Leukocyten sind, die Lymphocyten spärliche sind und an der Hornhaut keine typischen Follikeln bilden.

Das Trachoma verum corneae dagegen, das neugebildete Gewebe, ist reich an Follikeln. Die letzteren zeigen echte Keimzentren, die durch ihre Vereinigung grosse Granulationen und Tumoren entstehen lassen. Die Plasmazellen sind sehr selten zu finden und liegen meistens unter dem Epithel und zwischen den Keimzentren.

In dieser Hinsicht ist das Trachoma verum corneae dasselbe wie das Trachoma verum conjunctivae - es ist eine zusammenfliessende Conjunctivitis follicularis oder follikulare Hyperplasie der Bindehaut.

Wie das letztere, erleidet es dieselben Veränderungen: die kleinen Follikeln bilden durch ihre Vereinigung die Granulationen, welche nach Entleerung ihres Inhaltes degenerieren und in Cicatrisation uibergehen.

Der Grad der Entwicklung des Trachoma verum cornea ist, wie wir gesagt haben, bei Frühjahrskatarrh ganz individuell. Bei einigen Leuten bilden sich auf der Hornhaut trachomatöse Geschwülste, während die Bindehaut die Cicatrisation erleidet; bei andern entwickelt sich das Trachom mehr in dem Fornix als auf der Hornhaut usw.

Im Anfang entwickelt es sich an der Hornhaut zwischen dem Epithel und der Bowmanschen Membran, aber mit der Zeit nimmt es zu, helot das Epithel auf und kann tief in die Hornhaut eindringen und die Membrana Bowmani ganz zerstören - wie wir es bei einem. unserer Fälle beobachtet haben (V. Fall). In dieser Weise, nimmt es einen infiltrierenden Charakter und verhält sich wie die andere lymphadenoide Hyperplasien des Organismus.

Das Geschlecht spielt hierbei keine Rolle.

Was das Alter anbetrifft, so haben wir das Trachoma verum corneae bei jungen und alten Personen gefunden, sehr häufig bei Jünglingen.

Ausser diesem Merkmale besitzt das Trachoma verum corneae noch eins, das man verschieden zu erklären versucht hat, nämlich: dass es gewöhnlich am Limbus superior beginnt. Die Autoren, welche über den Pannus trachomatosus gearbeitet haben, haben um diesen sonderbaren Ursprung des Pannus trachomatosus zu erklären, verschiedene Hypothesen aufgestellt. Die einen haben diesen Ursprung der Injektion per contiguitatem zugeschrieben; die andern dem mechanischen Reiz. Es scheint uns, dass diese Hypothesen nicht ge- 
nügen, um uns diesen Ursprung des Trachoma verum comeae klar zu machen. Wir glauben, dass es sich um eine viel kompliziertere Ursache handelt. Die Ursache ist wahrscheinlich Schuld an zwei Sachen:

1. An der anatomischen Empfänglichkeit dieses Teiles des Limbus zur Bildung des lymphatischen Gewebes;

2. dass der Limbus superior sich in der Nachbarschaft des Fornix superior befindet, der reicher an Gefässen ist, und der die grössten trachomatösen Geschwülste bildet.

Die Ursache der Entstehung des Trachoma verum corneae ist dieselbe wie die der Bindehaut.

Durch die Beschreibung der charakteristischen Züge des Trachoma verum corneae in dem Vorhergehenden, haben wir erkannt, dass das Trachoma verum corneae keine einfache Entzündung ist. In der Tat, wenn die Entzündung das Resultat einer Reaktion des beschädigten Gewebes ist, so ist diese Reaktion in Trachoma verum corneae von solchen pathologischen. Veränderungen begleitet, die sich von den gewöhnlichen Veränderungen bei den echten entzündlichen Prozessen der Bindehaut sẹtr unterscheiden. Bei den letzteren bilden sich Follikeln und Papillen; während bei Trachoma verum die Anwesenheit von Follikeln nicht genügend ist das Trachom zu bilden; est ist noch etwas mehr nötig: der Zusammenfluss von diesen Follikeln. Trachoma verum ohne Follikeln gibt es nichti), aber jede Follikel ist nicht Trachom.

Auf der andern Seite haben wir gesehen, dass der Pannus selbst nicht dieselbe histologische Struktur hat. Es gibt Pannus mit zahlreichen, stark proliferierenden Plasmazellen, Pannus mit papillären Wucherungen - reich an Leukocyten und Pannus mit Follikeln und. zusammenfliessenden Keimzentren. Die ersteren haben wir besonders bei den akuten entzündlichen Prozessen kennen gelernt; der letzte ist für das Trachoma verum corneae bezeichnend.

Was ist also das Trachoma verum corneae?

Um das Wesen des Trachoma verum zu verstehen, muss man sich an das erinnern, was wir im vergangenen Jahre auf dem Ophthalmologenkongress zu Heidelberg über die Natur der Bindehaut gesagt haben.

Wir haben gesagt, dass trotz der viel umstrittenen Frage, ob die Bindehaut normalerweise Follikeln (oder lymphatischen Knötchen) hat

1) Es ist so wahr, dass wir haben die erste gezeigt, dass die Chlamidozoen nicht die Ursache des Trachoms sind, weil wir sie bei nicht follikularen Bindehautentzündungen gefunden haben. 
oder nicht, sie sich, im pathologischen Zustand, wie ein lymphatischer Apparat verhält. Dieser lymphatische Apparat kann sich hypertrophieren und erzeugt die Conjunctivitis follicularis oder die "Conjunctivitis granulosa acuta specifica (1), welche verschwinden kann, ohne eine Spur zu hinterlassen, oder in einen chronischen $\mathrm{Zu}-$ stand übergehen kann, wie dies der Fall ist bei allen lymphatischen Geweben und besonders bei den Ganglionen. Im andern Falle beginnt dieser Apparat zu wachsen, sei es durch eine vorläufige Reizung, wie zum Beispiel nach Conjunctivitis Gonorrhoica, sei es durch eine besondere lymphatische Empfänglichkeit ${ }^{2}$ ). In dieser Weise bildet sich ein neues lymphatisches Gewebe durch das Anwachsen und den Zusammenfluss der entwickelten Follikeln. Das Gewebe entsteht überall da, wo neue Gefässe sich bilden, und kann sogar die Hornhaut bedecken.

Dieser Hyperplasie des lymphatischen Apparates der Bindehaut haben wir den Namen - Trachoma verum gegeben.

Von diesem Standpunkt aus betrachtet, ist das Trachoma verum infolge seiner Bildung und seines Ursprungs nicht ein pathologischer Prozess, der ausschliesslich zur Bindehaut gehört, sondern allgemein und kommt auch bei andern Schleimhäuten, zum Beispiel der pharyngealen und nasopharyngealen vor.

Die Übereinstimmung im Ursprung, in der Bildungsweise, in der Evivolution und der Struktur, welche zwischen den Granulationen des Pharynx und denjenigen des Trachoma verum conjunctivae besteht, hat uns die Idee eingegeben, dass sie, sehr wahrscheinlich, auch dieselbe Ursache hat.

Wir sehen also, dass das Trachoma verum corneae, welches nichts anderes ist, als das Trachoma verum conjunctivae, eine Hyperplasie dieses selben Apparates sein muss, die sich vom Lïmbus über die Hornhaut ausbreitet.

Diese lymphadenoide Hyperplasie ist selten von einer allgemeinen ganglionären Reaktion begleitet, sondern ist vielmehr lokal. Nichtsdestoweniger ist seine Entwicklung, wie wir gesehen haben, ganz individuell, und dieses hängt, sehr wahrscheinlich, von einer lym phatischen Empfänglichkeit ab.

1) Prof. Eversbusch.

2) Wir haben letztens einen Fall beobachtet, wo echte trachomatöse Granulationen sich gebildet hatten, infolge einer Zahninfektion mit Oedema palpebrarum des Auges und grosser ganglionärer Hypertrophie und Eiterung. In der Familie litten mehrere Mitglieder an Amygdalitis hypertrophica und Vegetatio adenoides. 
Was die Behandlung des Trachoma verum comeae anbetrifft, so ist die Excision - die gründlichste und schnellste. Uber die Technik haben wir bereits vorn in der Arbeit berichtet.

Im Schluss möchten wir drei Tatsachen hervorheben, die ohne Zweifel in die Lehre des Trachoms eingeführt sein müssen.

Diese sind:

1. Neben dem Pannus fibrosus des Frühjahrskatarrhs, dem Pannus vasculosus simplex der vielen akuten Entzündungen der Bindehaut und Hornhaut, dem Pannus papillaris, dem Pannus plasmacellularis gibt es einen Pannus follicularis, der sich im Gegensatz zu Conjunctivitis follicularis durch den Zusammenfluss der Keimzentren und die Bildung geschwulstartiger Verdickungen reich an Keimzentren unterscheidet.

2. Diesem Pannus follicularis haben wir die Namen - Trachoma verum corneae gegeben.

3. Histologisch ist das Trachoma verum corneae von derselben Natur wie Trachoma verum conjunctivae und die lymphoide Granulationen und Wucherungen des Nasopharynx - es ist eine lymphadenoide - zusammenfliessende follikulare - Hyperplasie der Bindehaut.

\section{Literaturverzeichnis.}

1) Addario, Pannus trachomatosus. Ref. Arch. d'opht. 1911.

2) Bietti, Über Histologie des Pannus cornealis trachomatosus. Klin. Monatsbl. f. Augenheilk. 1908 .

3) Eversbusch, Die Augenerkrankungen im Kindesalter, 1912. S. 719.

4) Goldzieher, Beitrag zur pathologischen Anatomie des Trachoms. v. Graefe's Arch. f. Ophth. Bd. LXIIT. S. 287.

5) Ichikawa, Beitrag zur Trachomfrage. v. Graefe's Arch.f. Ophth. Ba.LXXIII. S. 303 .

- Trachomatöse Veränderungen der Skleralbindehaut. Ebenda. Bd. LXXIX. S. 64 .

6) Komoto, Über die Histologie des Pannus trachomatosus. Klin. Monatsbl. f. Augenheilk. 1909.

7) Megardi, Sull' Istologia del panno corneale trachomatoso. Arch. di Ottalm. XIV. p. 185.

8) Meyerhof, Follikelbildung im Pannus trachomatosus. Klin. Monatsbl. $f$ Augenheilk. 1908.

9) Muttermilch, Uber die Ätiologie und das Wesen des Trachoms. $\mathrm{v} . \mathrm{Graefe}$ Arch. f. Ophth. Bd. LXXIII. S. 384.

10) Nuel, System of Diseases of the Eye. T. IV. p. 199. Norris \& Oliver.

11) Pascheff, Le Catarrbe printanier. Arch. d'opht. 1907.

- Recherches sur la nature et l'etiologie da yrai trachom. Arch. d'opht. 1910.

- Plasmazellenbildungen der Bindehaut und Hornhaut. y. Graefe's Arch. f. Ophth. Bd. LXVIII u. LXXIII.

- Hämatopoetische Funktion der Bindehaut. Ophth. Ges. zu Heidelberg. 1911. Arch. f. Hämatologie. 1911. 
Pascheff, Medizin. Kongress zu Budapest. 1909.

- Über die Chlamydozoen und Trachomkörperchen. v. Graefe's Areh. f. Ophth. Bd. LXXIII.

12) Rählmann, Histologischer Bau des Pannus trachomatosus. v. Graefe's Arch. f. Ophth. 1897.

1.3) Römer, Lehrbuch der Augenheilkunde. 1913.

14) Stanculeanu u. Michail, Das Trachorn nach dem gegenwärtigen Stand der Forschung.

Erklörung der Abbildungen auf Taf. XVI u. XVII, Fig. 1-31.

Fig. 1. Isolierte und zusammenfliessende Follikeln in der Nähe der oberen Fornix bei Trachoma verum conjunctivae sclerae.

Fig. 2. Bildung von Granulationen bei Trachoma verum durch den $\mathrm{Zu}$ sammenfiuss der Follikeln. nices.

Fig. 3. Weitere Bildung von grosser Granulation bei. Trachoma verum for-

Fig. 4, 5 und 6. Isolierte und zusammenfliessende Follikeln in der Umgebung der Gefässe bei Trachoma conjunctivae sclerae.

Fig. 7. Einzelne grosse Granulation der Conjunctivae sclerae.

Fig. 8. Follikeln des Fornices und Conjunctiva sclerae bei Trachoma verum.

Fig. 9. Grosse Granulation Conjunctivae sclerae.

Fig. 10. Granulationen um die Gefässe herum der Conjunctiva sclerae bei Atrophia bulbi $(a, b)$.

Fig. 11. Trachoma verum comeae oder Pannus follicularis. Fall 1.

Fig. 12. Einzelne Granulation der Hornhaut.

Fig. 13. Trachoma verum (follicular.) corneae. Fall XI.

Fig. 14. Pannus vasculosus simplex bei Trachoma verum mit Follikel Conjunctivae sclerae in der Năhe des Limbus superior corneae. Fall XIII.

Fig. 15. Trachoma verum corneae (Pannus follicularis). Fall II.

Fig. 16. Trachoma verum corneae. Fall XII.

Fig. 17. Trachoma verum corneae. Fall VIII.

Fig. 18. Trachoma verum corneae und Conjunctivae sclerae. Fall VI.

Fig. 19. Trachoma verum corneae (Geschwulst). Fall VI.

Fig: 20. Trachoma verum corneae. Fall IX.

Fig. 21. Vertikaler Schnitt parallel zum horizontalen Meridian der Hornhaut. Fall XIII. Vergr. 16/1.

Fig. 22. Vertikaler Schnitt parallel zu dem horizontalen Meridian der Hornhaut. Fall XI. Vergr. 16/1.

Fig. 23. Vertikaler Schnitt parallel zu dem horizontalen Meridian der Hornhaut. Fall VIII. Vergr. 16/1.

Fig. 24. Derselbe Sehnitt bei Fall I. Vergr. 16/1.

Fig. 25. Derselbe Schnitt bei Fall III. Vergr. 10/1.

Fig. 26. Vertikaler Schnitt parallel zu dem vertikalen Meridian der Hornhaut. Fall V. Vergr. 16/1.

Fig. 27. Vertikaler Schnitt parallel zu dem horizontalen Meridian der Hornhaut. Derselbe Fall. Vergr. 16/1.

Fig. 28. Vertikaler Schnitt parallel zu dem horizontalen Meridian der Hornhaut. Fall IV. Vergr. 16/1.

Fig. 29. Derselbe Fall und Schnitt. Zwei Keimzentren getrennt durch Epithelsprosse. Vergr. 50/1.

Fig. 30. Vertikaler Schnitt parallel zu dem vertikalen Meridian der Hornhaut. Fall VI.

Fig. 31. Pannus simplex bei Trachoma verum conjunctivae mit Pterygium reich an Follikeln. 
v. Graefe's Archiv. Bd. LXXXVII.
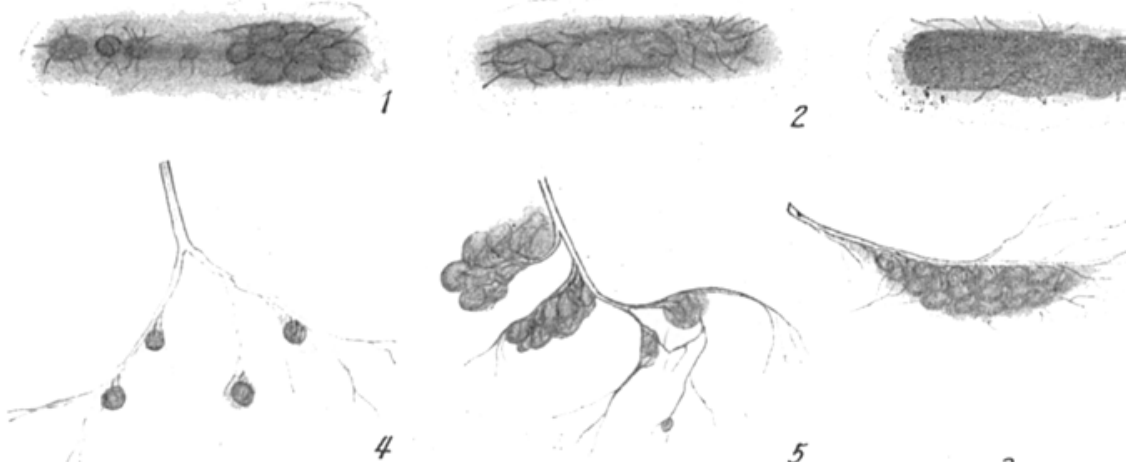

6

5
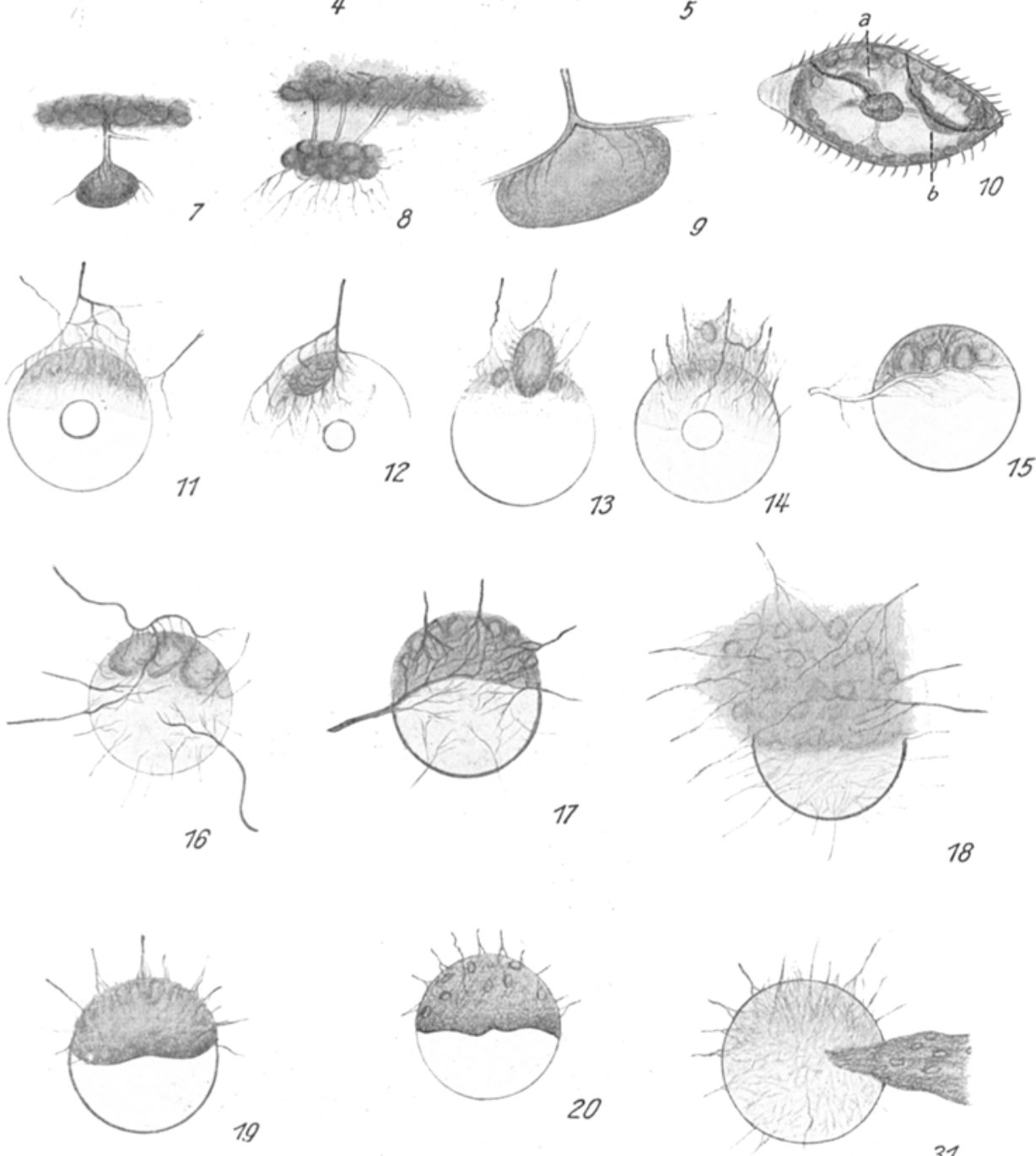
Taf. XVI.

a

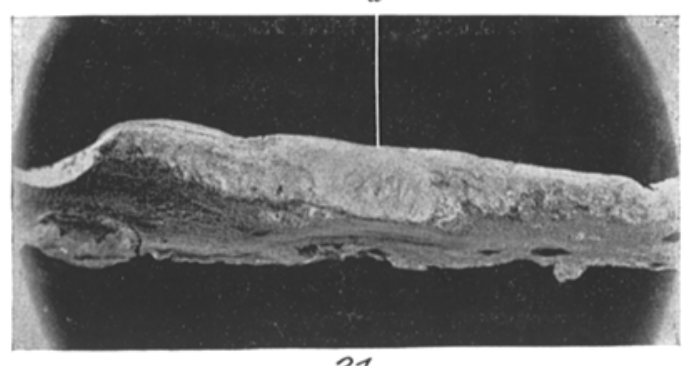

21

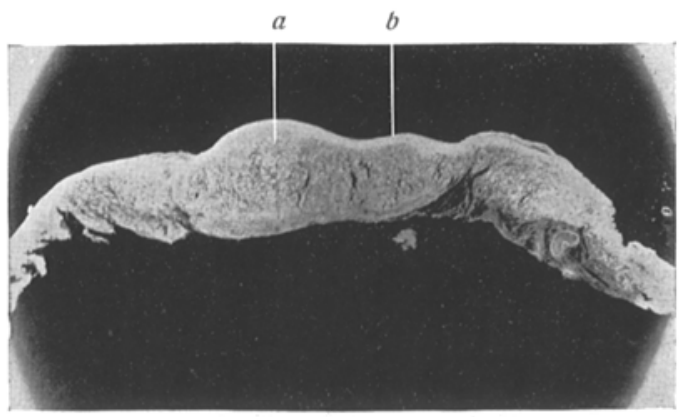

22

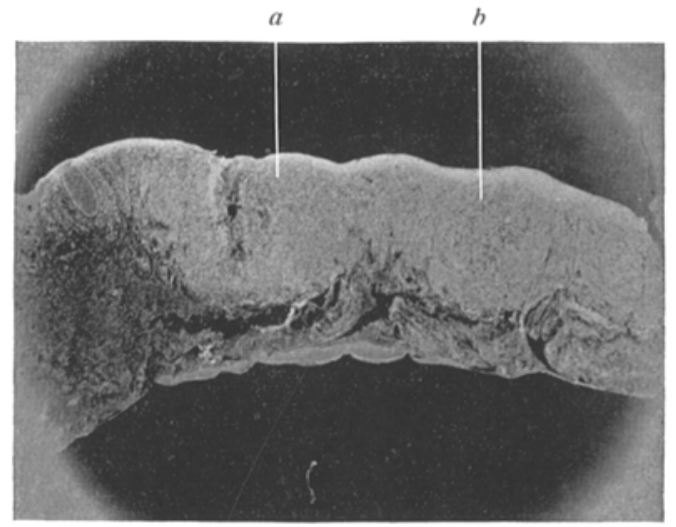

23

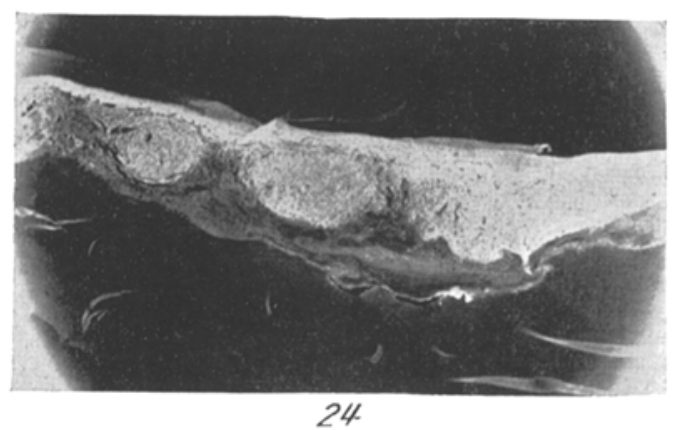

inn in Leipzig und Berlin. 


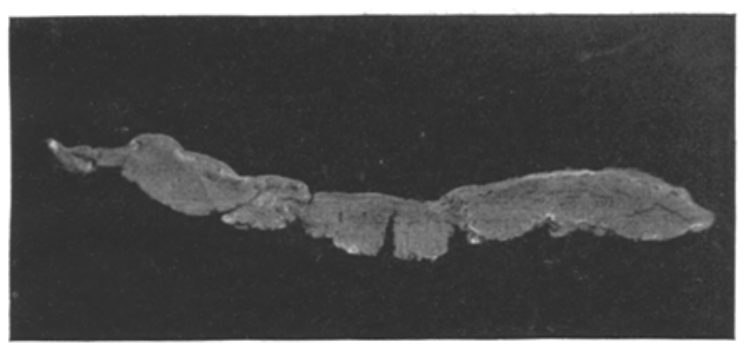

25

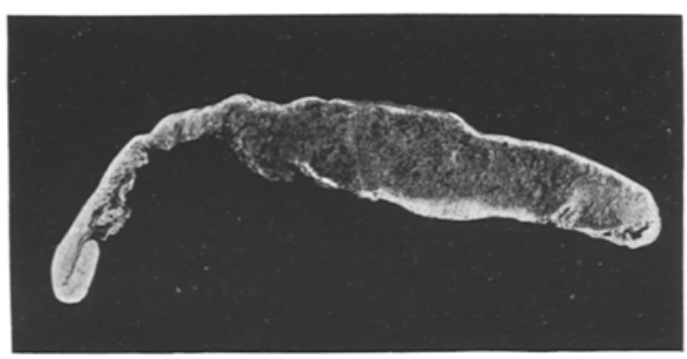

26

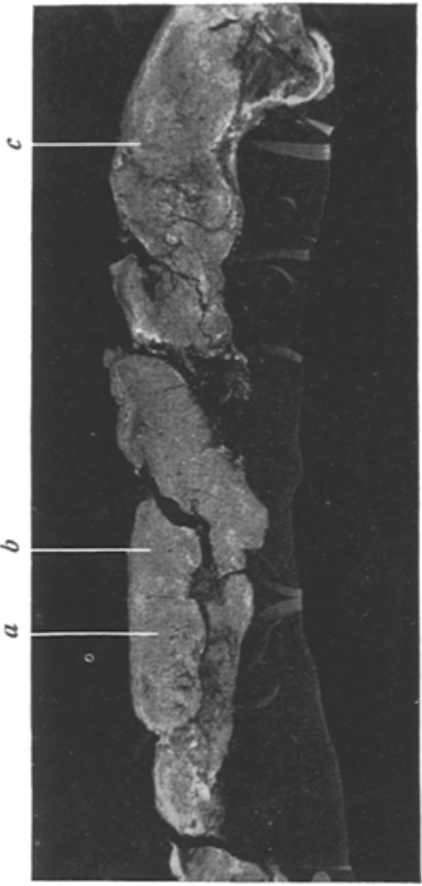

28
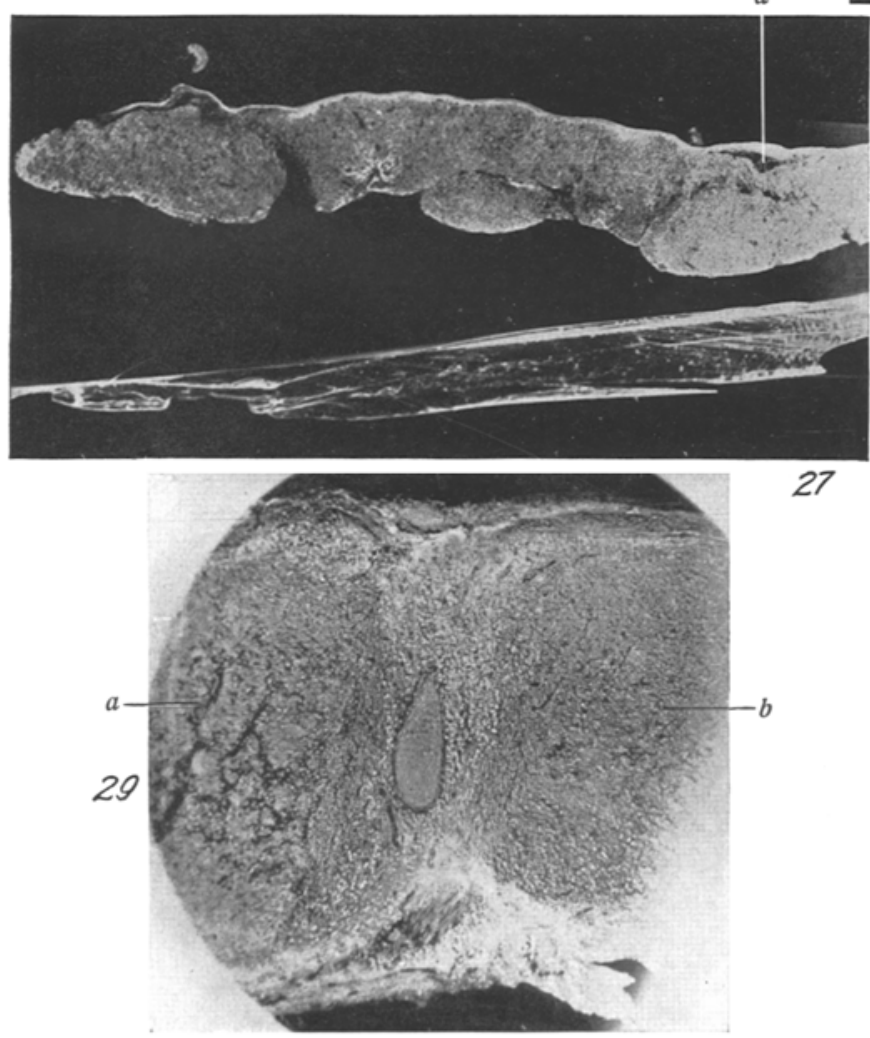

27

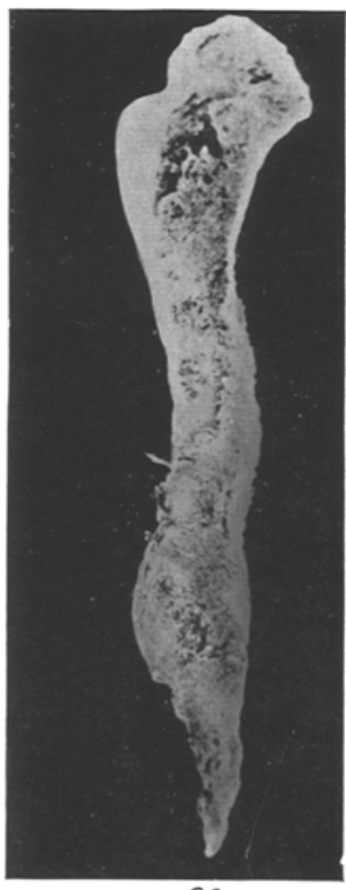

30

Veriag von Wilhelm Engelmann in Leipzig und Berlin. 